\title{
Annotations
}

\section{Nursing sick children}

'Most children's wards are without a children's trained nurse for some of the time, many are without for much of the time, and some are without at all times'.

It is desirable, reasonable, and increasingly necessary that permanent nursing staff on children's wards should have a recognised qualification for, or extensive experience of, nursing children. The complexity of care has increased considerably during the last few years with the introduction of intensive care for neonates and specialisation within paediatrics, although the number of children admitted to hospital with acute infections has decreased. Domiciliary care of sick children previously admitted to hospital has increased and there is a need for paediatric nursing skills in the district nursing service. At times, untrained nursing staff find the strain of nursing sick children unbearable; an observation made by Florence Nightingale when she recommended that children should be admitted to women's wards so that the other patients could help with the care. In 1959 the Platt report ${ }^{2}$ recommended that the sister-in-charge of a children's ward should be both a Registered Sick Children's Nurse (RSCN) and a State Registered Nurse (SRN). Since then, other grades of nursing staff have been created-such as the State Enrolled Nurse (SEN) which is a general qualification although some nurses proceed to a special children's training via a Joint Board Course of Nursing Studies. Other nursing staff on a children's ward may include nursery nurses, nursing auxiliaries, and students and pupils undertaking training for the SRN or SEN qualification, or postregistration training for the RSCN. The proportion of all learners to trained staff is controlled according to the district policy and the district budget; the requirement to train SRN students may limit the number of RSCN learners that can be accommodated.

In 1971 the Department of Health and Social Security (DHSS) ${ }^{3}$ reiterated previous recommendations concerning children in hospital (Platt report, HM (59) 19, HM (62) 39, HM (66) 18, HM (69) 4), and stated that sisters-in-charge of a children's ward should have an RSCN qualification and that all staff on the ward should receive special training in caring for children. Moreover it emphasised that the nurse : patient ratio in a children's ward should be higher than in a comparable adult ward and should include a greater proportion of trained nurses.

The various reports and circulars have made little impact: in 1975 only $20-50 \%$ of trained staff were RSCN, with wide regional variations, and $15 \%$ of wards had no trained staff by day, $50 \%$ of them had no trained staff at night, and $11 \%$ of them had no trained staff at any time. A recent survey ${ }^{4}$ concentrating on training facilities found that $37 \%$ of staff nurses and $18 \%$ of sisters on children's wards did not have the RSCN qualification. The same survey showed that $5 \%$ of established sisters' posts and $12 \%$ of staff nurses' posts were unfilled because of failure to recruit. It is difficult to devise a realistic formula for determining the proper nursing establishment for children's wards because the requirements vary widely according to the type of work carried out. Numerous reports of inadequate establishments, especially at night, have been received by the British Paediatric Association and British Association of Paediatric Surgeons (BPA/ BAPS) nursing committee, and the complexity of the work in some units has increased the need for trained staff. In 1973 the establishment for RSCNs in a survey of three health authority regions was found to be just over half that considered desirable. ${ }^{1}$ Clearly, new information regarding the adequacy of the nursing establishment for children and the availability of RSCNs is required. HC (77) 30 required Regional Health Authorities (RHAs) to provide the DHSS with information about their hospital facilities for children by December 1977, and this might have helped; perhaps those RHAs which did respond did so in such a way that the information was indigestible because no report has appeared. Accordingly, the BPA/BAPS nursing committee is now conducting a further survey to obtain this vital information from members throughout the UK, and it is hoped that all will respond.

Since 1972 an average of 617 RSCNs have qualified annually, with variations between 549 in 1978 and 647 in 1979. During this period the training courses for RSCN alone have been largely stopped in England and Wales (although not in Scotland) and have been replaced by an increase in the 13-month postbasic RSCN course for SRNs, while the numbers completing the combined RSCN/SRN course have remained relatively constant. ${ }^{4}$ Overall the numbers have not fallen, as was feared, but nor have they 


\section{Chantler}

increased to improve the known deficiencies. The fluctuation in the numbers starting training and qualifying year-by-year is wide. For instance, between April and September 1980, 413 nurses qualified as RSCN (a reduction of $6 \%$ ), whereas in the same period 333 started courses for the combined RSCN/SRN qualification or for the 13-month postbasic RSCN course (a rise of $17 \%$ ). A wide regional variation is apparent. The mean provision of RSCN places each year for England and Wales is 19 per million population, whereas training places for each region vary from 0 to 48 places per million population, even excluding The Hospital for Sick Children, Great Ormond Street, London, which offers 230 places each year of a total of 905 places available nationally. The number of places available is entirely under the control of the district nursing officers because the RHAs and DHSS can only advise and this advice is clearly not very effective. District plans and budgets are affected by many considerations, among which the regional need for paediatric nursing is unlikely to feature prominently. Moreover, the new EEC directives and the new General Nursing Council (GNC) syllabus for RSCN training, which requires more community experience for learners, together with financial stringency, are likely also to reduce the number of places available.

There is no evidence of a shortage of people applying to fill places ${ }^{4}$ and the numbers who fail to complete courses are not excessive compared with other branches of nursing. There is concern about the number of RSCNs who do not continue to work with children, and the DHSS is carrying out a research study to find out why, and to enquire into the career prospects for RSCNs. This study, while admirable and worthwhile, is being used as an excuse to avoid effective positive action by the DHSS to increase the number of RSCNs. The recent decision of the staff side of the Whitley Council to press for a pay lead for RSCNs working with children, together with other specialised nursing groups, is encouraging but unlikely to lead to any material result as long as the staff side continues simply to divide the percentage pay rises among all concerned rather than giving more to areas of shortage; presumably this is because of their dislike of differentials.

What then can be done to correct this sorry state? The BPA/BAPS joint committee on nursing has made the following recommendations which have been endorsed by its parent bodies.

(1) All children's ward sisters appointed after a predetermined date should hold the RSCN qualification. (2) RHAs should be responsible for determining regional needs and funding training places. The DHSS should be responsible for a yearly review of regional plans and national requirements.
(3) The 13-month postbasic SRN course for RSCN should be increased by establishing new courses and enlarging existing ones. The GNC should encourage and support the introduction of experimental courses-such as the 6-month course for RSCN for SRNs who already have extensive experience with children. The paediatric content of the SRN course should be reviewed to reduce the stress on young students by increasing the amount of experience gained in the community with healthy children. This will enable more learner places on children's wards to be occupied by RSCN students.

(4) The financial lead for RSCNs should be introduced as soon as possible.

These recommendations deserve to be supported by all concerned. In particular, the responsibility of the RHA and DHSS should be acknowledged. Central funding from the DHSS for teachers of midwifery, health visiting, and district nursing has now been agreed and central funding of nurse tutors organised through the GNC is a long-standing arrangement. Central or regional funding of the salaries of RSCN trainees could be regarded as an extension of this precedent. The GNC should examine the training arrangements and career prospects and encourage recruitment of paediatric nurse tutors. An urgent examination of the career prospects of RSCNs should be undertaken by the Royal College of Nursing, the Association of British Paediatric Nurses, and the DHSS when the results of the DHSS survey of nurses with the RSCN qualification are complete. The DHSS has repeatedly advised that all children in hospital should be accommodated in children's wards and this should with rare exceptions be mandatory; as well as desirable in itself it would increase the opportunities for designing satisfactory RSCN training courses. Twenty-one years after Platt, children are still not being properly nursed in British hospitals and the government should insist on action now.

\section{References \\ 1 Court S D M, chairman. Fit for the future. Report of the Committee on Child Health Services. Vol. 1. London: HMSO, 1976: 320. \\ 2 Platt H, chairman. The welfare of children in hospital. Great Britain Central Health Services Council. London: HMSO, 1959. \\ 3 Hospital facilities for children. Hospital Memoranda. DHSS HM (71) 22: 1971. \\ 4 BPA/BAPS Joint Committee on Nursing. Children's nurses. A survey. London: British Paediatric Association,} 1980.

Cyril Chantler Evelina Department of Paediatrics, Guy's Hospital, St Thomas Street, London SE1 9RT 